\title{
Return to Sport in Athletes with Midportion Achilles Tendinopathy: A Qualitative Systematic Review Regarding Definitions and Criteria
}

\author{
Bas Habets ${ }^{1,2}$ (D) Anke G. van den Broek ${ }^{2}$ Bionka M. A. Huisstede ${ }^{2}$ • \\ Frank J. G. Backx ${ }^{2} \cdot$ Robert E. H. van Cingel ${ }^{1,3}$
}

Published online: 16 December 2017

(C) The Author(s) 2017. This article is an open access publication

\begin{abstract}
Background Midportion Achilles tendinopathy (AT) can cause long-term absence from sports participation, and shows high recurrence rates. It is important that the decision to return to sport (RTS) is made carefully, based on sharply delimited criteria. Lack of a well-defined definition and criteria hampers the decision to RTS among athletes with AT, and impedes comparison of RTS rates between different studies.

Objective The aim of this study was to systematically review the literature for definitions of, and criteria for, RTS in AT research.

Study Design Qualitative systematic review.

Methods The PubMed, EMBASE, Cochrane, CINAHL, PEDro, and Scopus electronic databases were searched for articles that reported on the effect of a physiotherapeutic intervention for midportion AT. Article selection was independently performed by two researchers. Qualitative content analysis was used to analyze the included studies and extract definitions of, and criteria for, RTS.
\end{abstract}

Electronic supplementary material The online version of this article (https://doi.org/10.1007/s40279-017-0833-9) contains supplementary material, which is available to authorized users.

Bas Habets

b.habets@umcutrecht.nl

1 Papendal Sports Medical Centre, Papendallaan 7, 6816 VD Arnhem, The Netherlands

2 Department of Rehabilitation, Physical Therapy Science and Sports, Rudolf Magnus Institute of Neurosciences, University Medical Center Utrecht, Utrecht, The Netherlands

3 Radboud University Medical Centre, Research Institute for Health Sciences, IQ Healthcare, Nijmegen, The Netherlands
Results Thirty-five studies were included in the content analysis, showing large variety in both the definitions and criteria. Thirty-two studies reported a definition of RTS, but only 19 studies described the criteria for RTS. The content analysis revealed that 'reaching pre-injury activity/ sports level, with the ability to perform training and matches without limitations', 'absence of pain', and 'recovery' were the main content categories used to define RTS. Regarding the criteria for RTS, eight different content categories were defined: (1) 'level of pain'; (2) 'level of functional recovery'; (3) 'recovery of muscle strength'; (4) 'recovery of range of motion'; (5) 'level of endurance of the involved limb'; (6) 'medical advice'; (7) 'psychosocial factors'; and (8) 'anatomical/physiological properties of the musculotendinous complex'. Many criteria were not clearly operationalized and lacked specific information.

Conclusions This systematic review shows that RTS may be defined according to the pre-injury level of sports (including both training and matches), but also with terms related to the absence of pain and recovery. Multiple criteria for RTS were found, which were all related to level of pain, level of functional recovery, muscular strength, range of motion, endurance, medical advice, psychosocial factors, or anatomical/physiological properties of the Achilles tendon. For most of the criteria we identified, no clear operationalization was given, which limits their validity and practical usability. Further research on how RTS after midportion AT should be defined, and which criteria should be used, is warranted.

PROSPERO Registration Number CRD42017062518. 


\section{Key Points}

There appears to be large variation in how return to sport (RTS) after midportion Achilles tendinopathy (AT) is defined within the current literature.

Numerous criteria for RTS are proposed, but the majority of these criteria lack clear operationalization and cut-off values.

There is a strong need for clinicians and researchers to reach consensus on a clear definition and criteria for RTS after midportion AT.

\section{Introduction}

Midportion Achilles tendinopathy (AT) can cause a prolonged absence from sports participation, and may even be career-ending in up to $5 \%$ of athletes with AT [1]. Recurrence rates as high as $27 \%$ have been reported, particularly in those with short recovery periods ( $0-10$ days) [2], which might be related to the fact that, although symptoms have fully subsided, deficits in musculotendinous function may still persist in $25 \%$ of patients, putting the athlete at risk for re-injury [3]. Therefore, it is important that a decision on return to sport (RTS) is carefully made, based on multiple factors and involving all relevant stakeholders [4].

In a recent systematic review on eccentric training for midportion AT, performed by our research group [5], we found that only one-third of the included studies used RTS as an outcome, with a RTS rate ranging between 10 and $86 \%$ after 12 weeks $[6,7]$. These studies used different definitions (e.g. 'return to previous activity level' or 'return to full activity'), which makes comparison of their RTS rates difficult. In many other AT studies, RTS is either not the main outcome of the study or is not evaluated at all. This results in a lack of clear definition of RTS and an absence of well-defined criteria for RTS.

In 2016, a consensus statement on RTS after sports injuries was developed [4] which stated that "the definition of each RTS process should, at a minimum, be according to the sport $[\ldots]$ and the level of participation [...] that the athlete aims to return to" [4]. Silbernagel and Crossley [8] recently proposed a program aimed at RTS for athletes with midportion AT. While this program provides a useful rationale and progression to RTS, unfortunately the authors did not explicitly report a single clear definition of RTS, or the exact criteria that should be met.
The lack of a clear definition and well-defined criteria can hamper the decision making for RTS among athletes with AT. Moreover, it impedes comparison of RTS rates between different intervention studies. Therefore, the aim of this review was to systematically analyze the current literature for definitions of RTS in AT research, and investigate which criteria for RTS are being used.

\section{Methods}

\subsection{Study Design}

This systematic review was developed in accordance with the Preferred Reporting Items for Systematic Reviews and Meta-Analyses (PRISMA) guidelines, and was prospectively registered in the PROSPERO database for systematic reviews (registration number CRD42017062518).

The purpose of the study was twofold: (1) to synthesize definitions of RTS, where RTS was seen as a successful endpoint after midportion AT; and (2) to search for criteria used in scientific literature for decision making to initiate RTS.

\subsection{Search Strategy}

A systematic search of the literature from 1998 to July 2017 was conducted in the PubMed, EMBASE, Cochrane, CINAHL, PEDro, and Scopus electronic databases. The search was limited from 1998 onwards based on the paper from Maffulli et al. [9]. According to this paper, the terminology changed from 'tendinitis', considered as a frank inflammation of the tendon, to 'tendinopathy', which is a combination of frequent longstanding pain, swelling and impaired performance [9]. This paradigm shift has led to changes in the management of tendinopathic injuries (i.e. targeted more at reducing symptoms and increasing load capacity rather than minimizing inflammation using nonsteroid medication and/or injections), and this can have consequences for the factors associated with the RTS decision.

The search strategy contained various synonyms for 'Achilles tendinopathy'. For 'return to sport', we partially adopted a search strategy used in a similar research on return to play after hamstring injuries [10], and modified this to fit our study purpose. The final search strategy can be found in electronic supplementary Appendix S1.

\subsection{Eligibility Criteria}

All retrieved articles were independently screened for eligibility by two authors (BHa, AvdB). All studies investigating the effect of any physiotherapeutic intervention in 


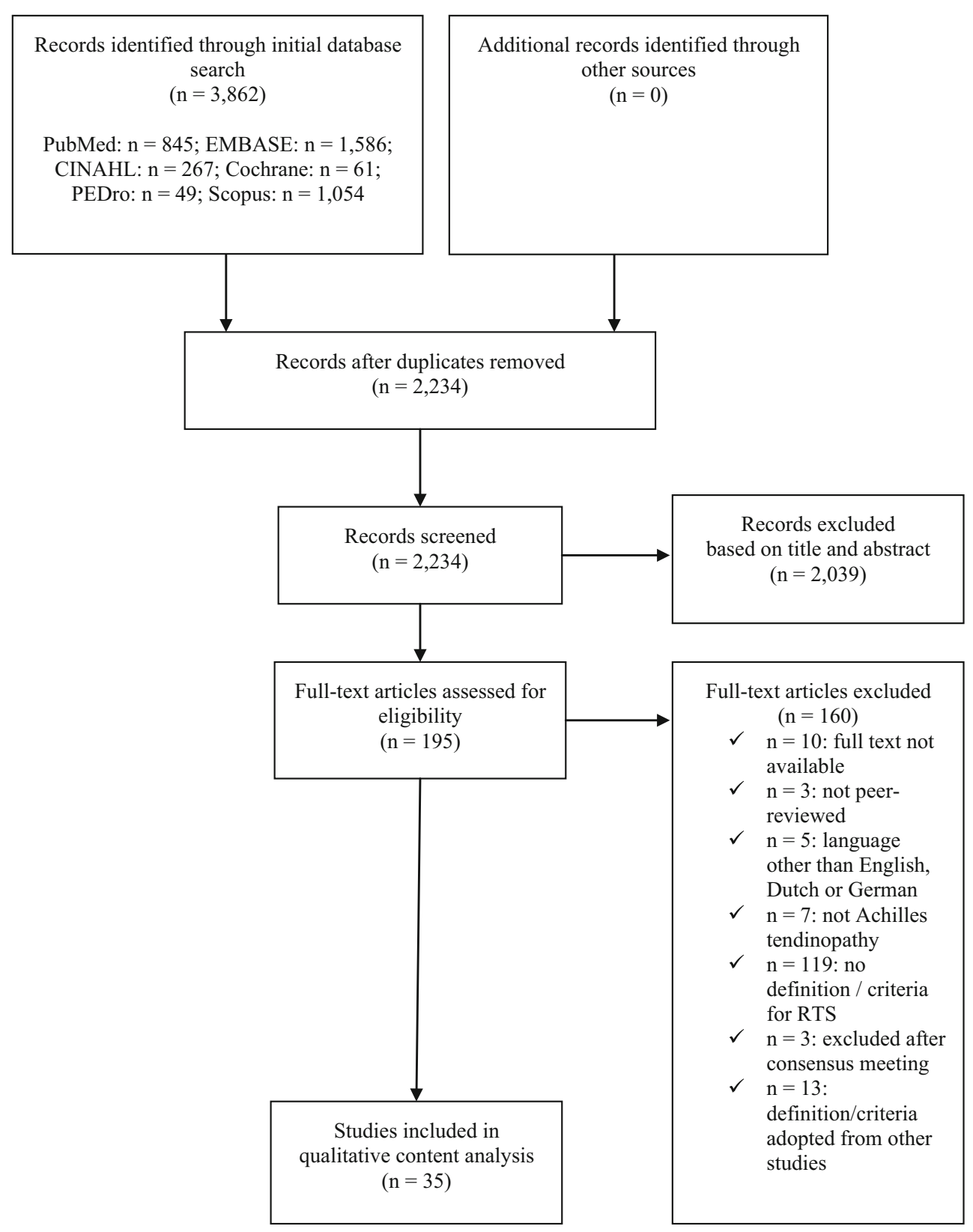

Fig. 1 Study search strategy. RTS return to sport

an adult ( $\geq 18$ years) athletic population (i.e. individuals who participate in organized or non-organized sports) with midportion AT were eligible for inclusion if they (1) described a definition of, and/or criteria for, RTS, and (2) were written in English, Dutch or German. There were no restrictions on type of study design. Articles that adopted definitions from other studies were excluded, but the studies from which the original definition was adopted were screened for eligibility, and included when they met our eligibility criteria. Potential articles were further excluded if they (1) were not available in full-text, despite serious efforts to contact the corresponding author; (2) described interventions for insertional AT and/or Achilles tendon rupture; (3) investigated surgical or other invasive interventions; or (4) were animal studies.

A consensus meeting between the two authors was held to discuss discrepancies in article screening and selection. If no consensus could be reached between the two authors, a third author $(\mathrm{BHu})$ was asked to make a final decision. Cohen's kappa was calculated to indicate agreement 


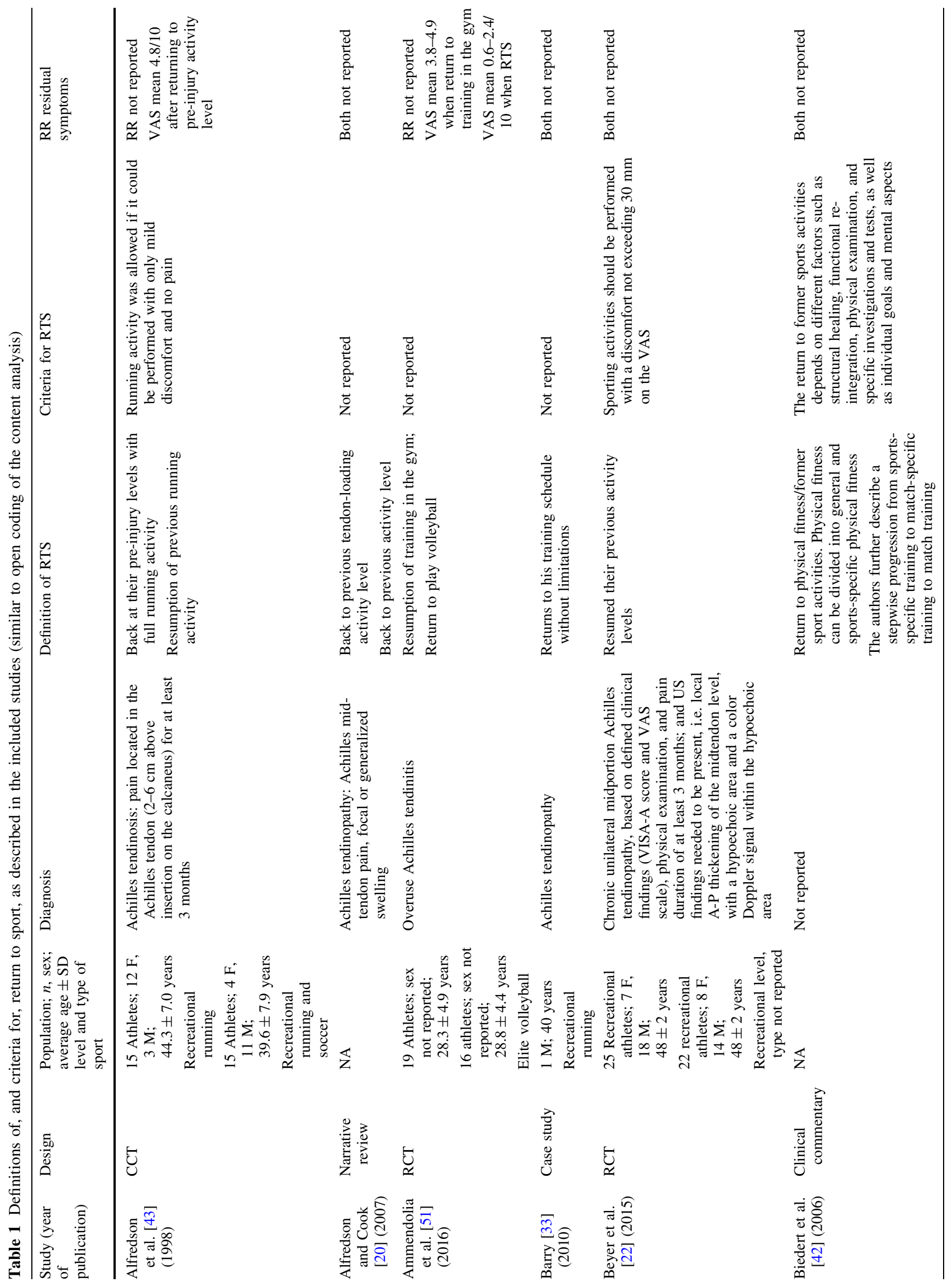




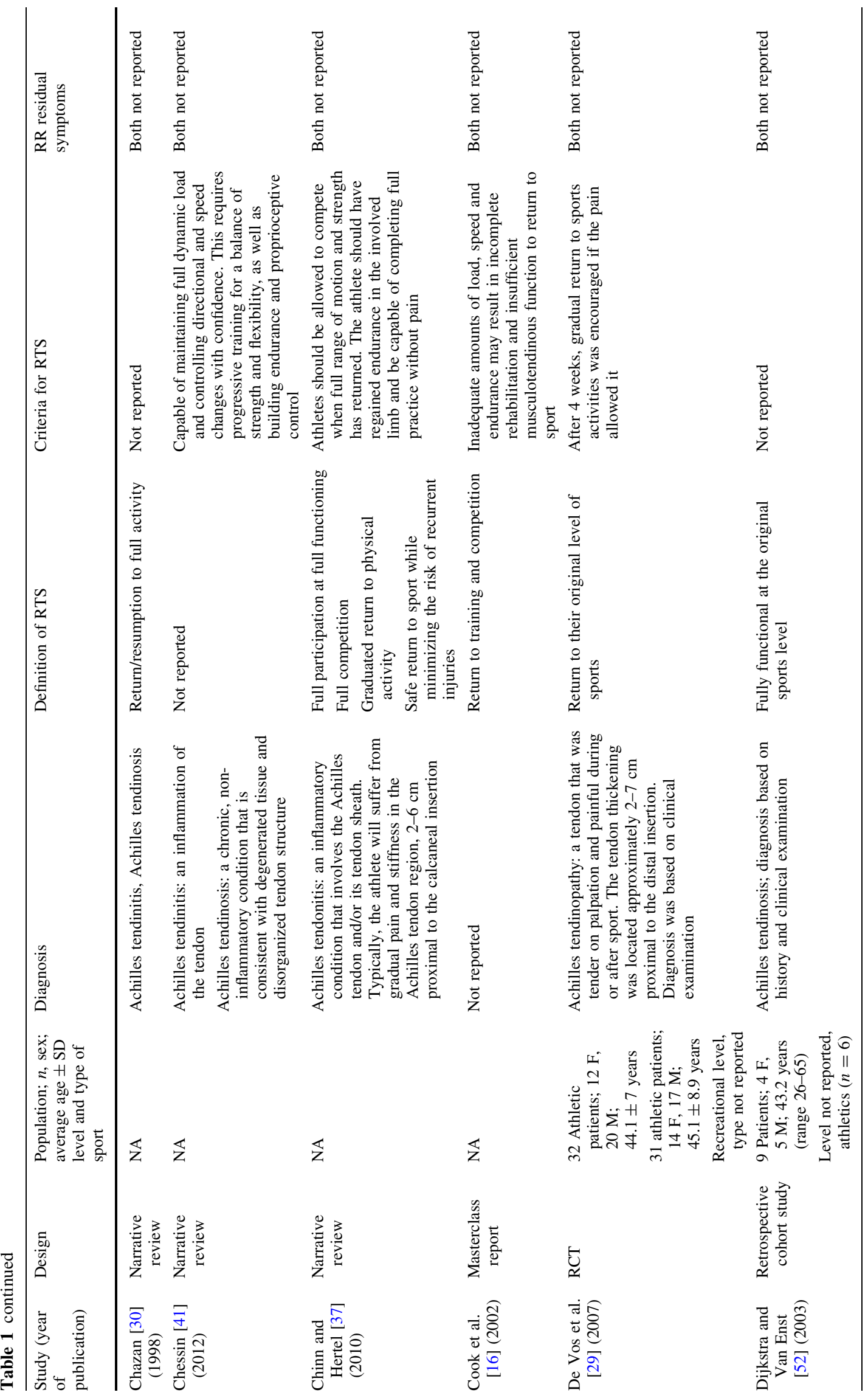




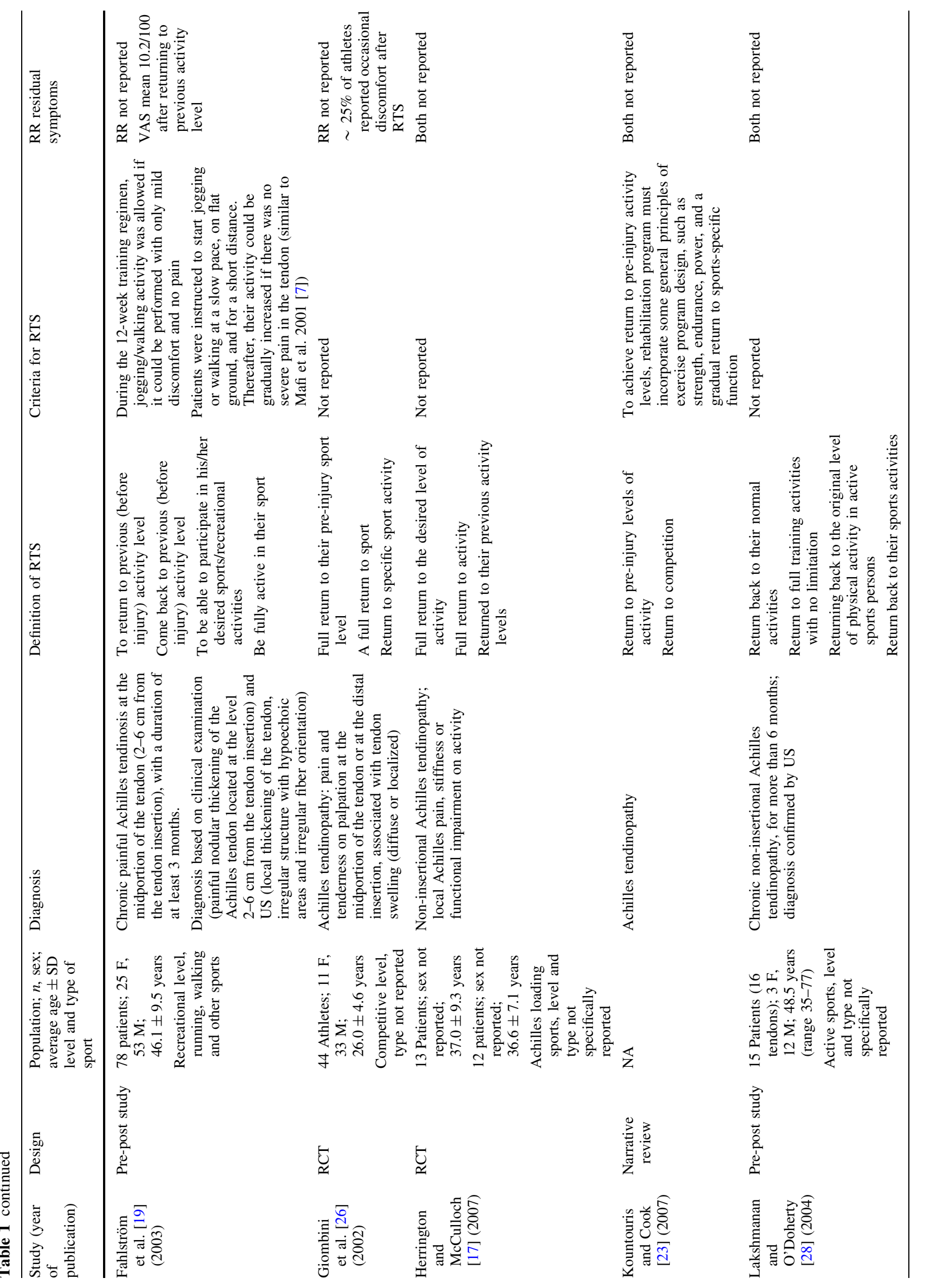




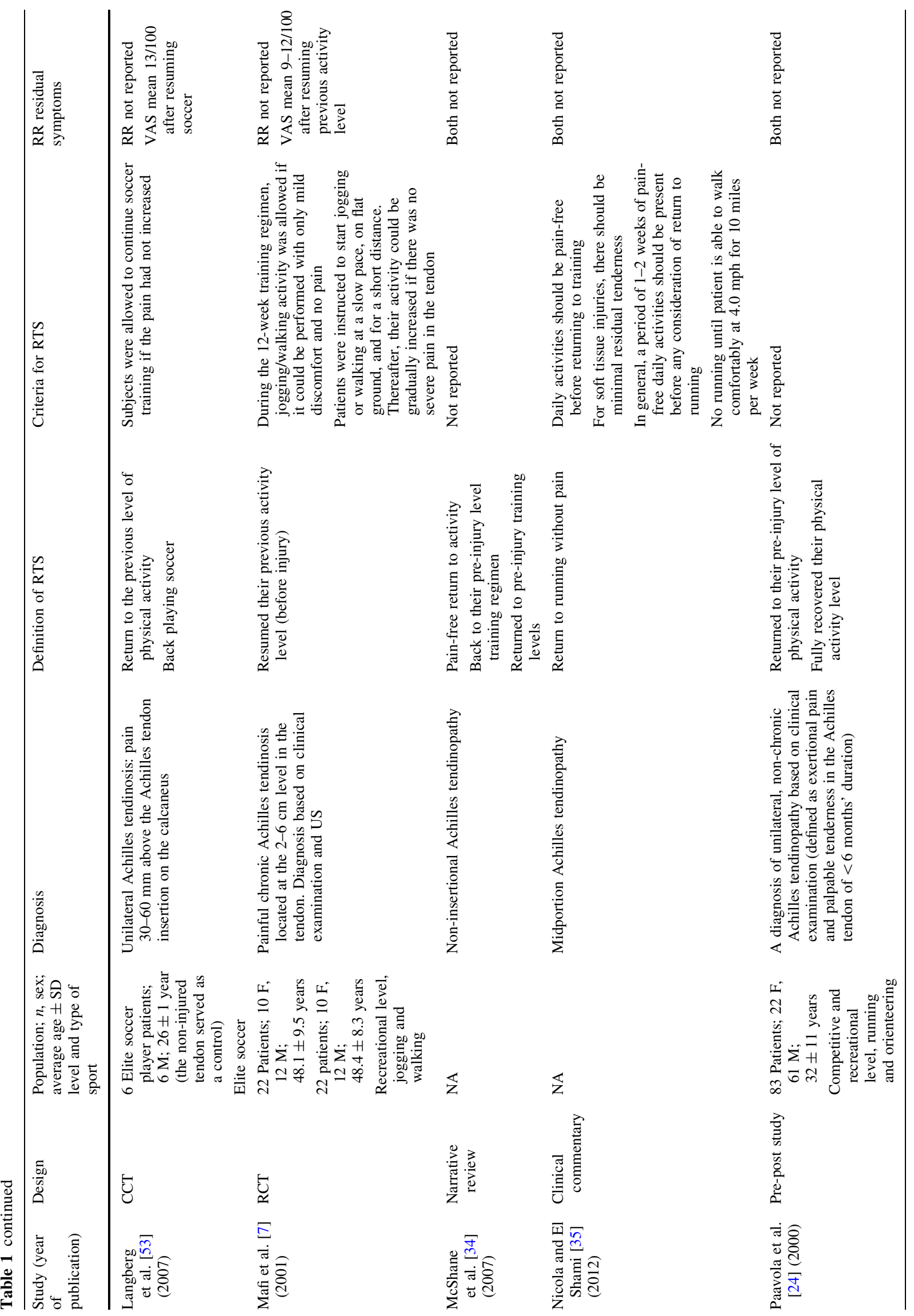




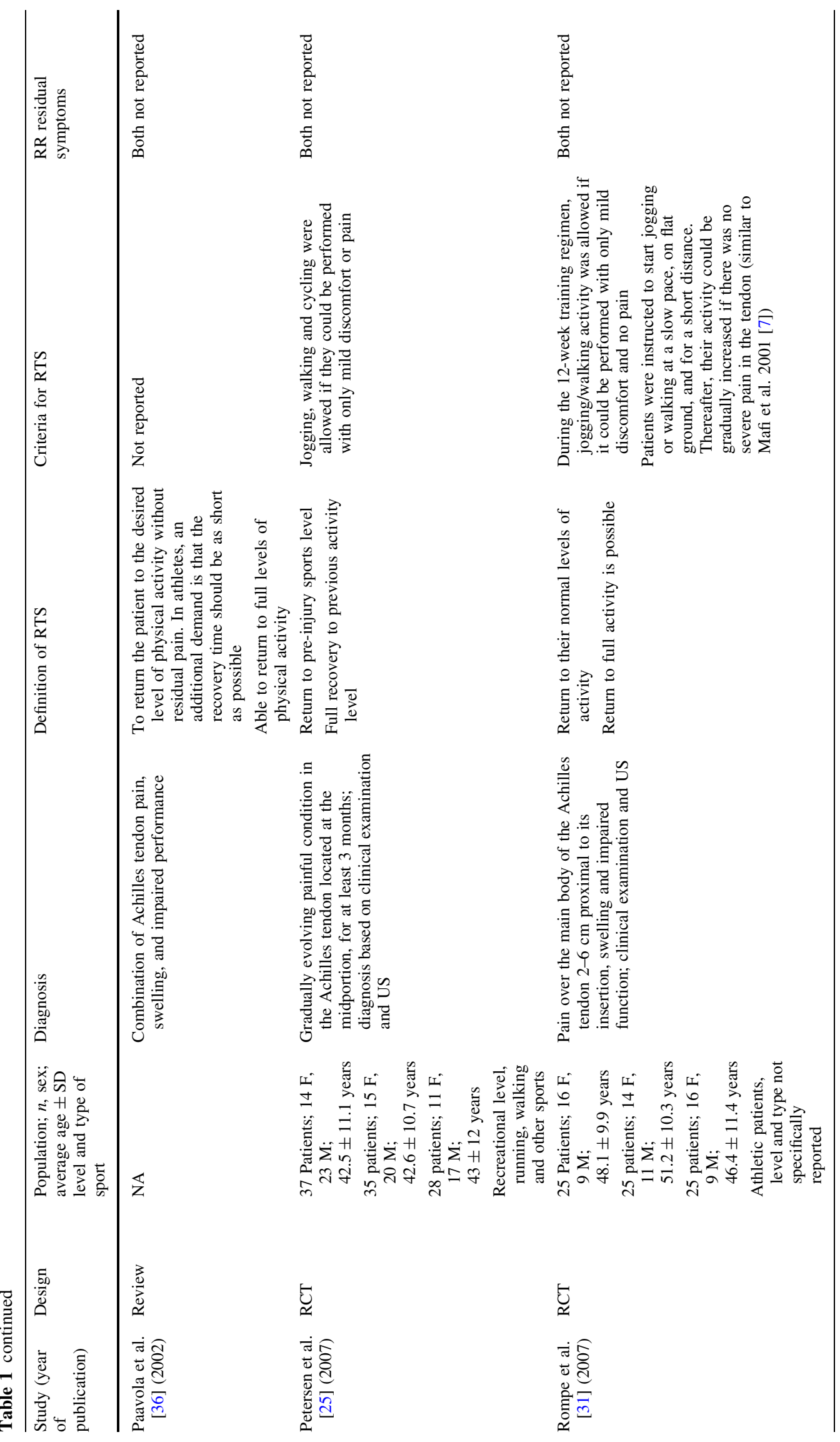




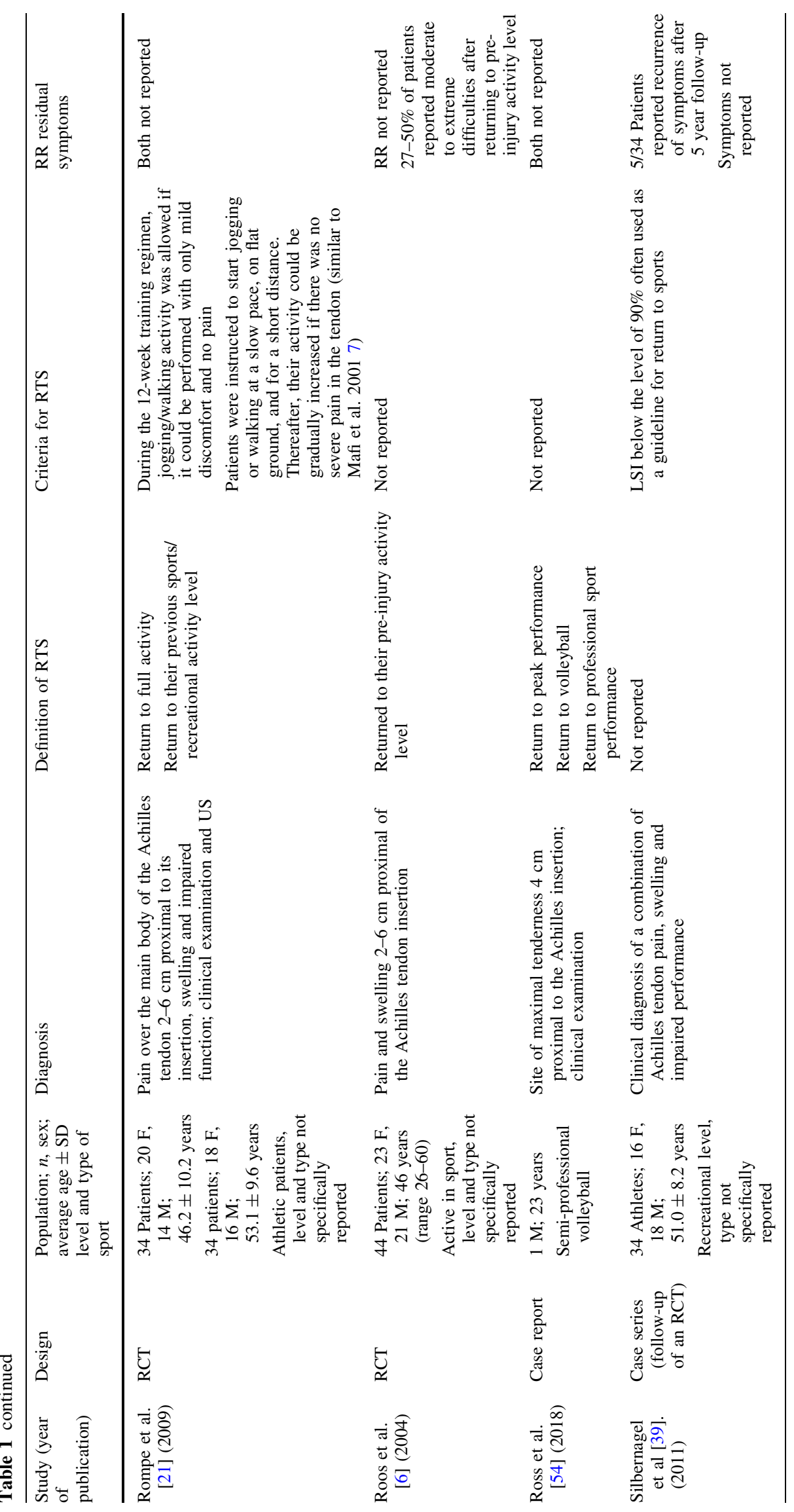




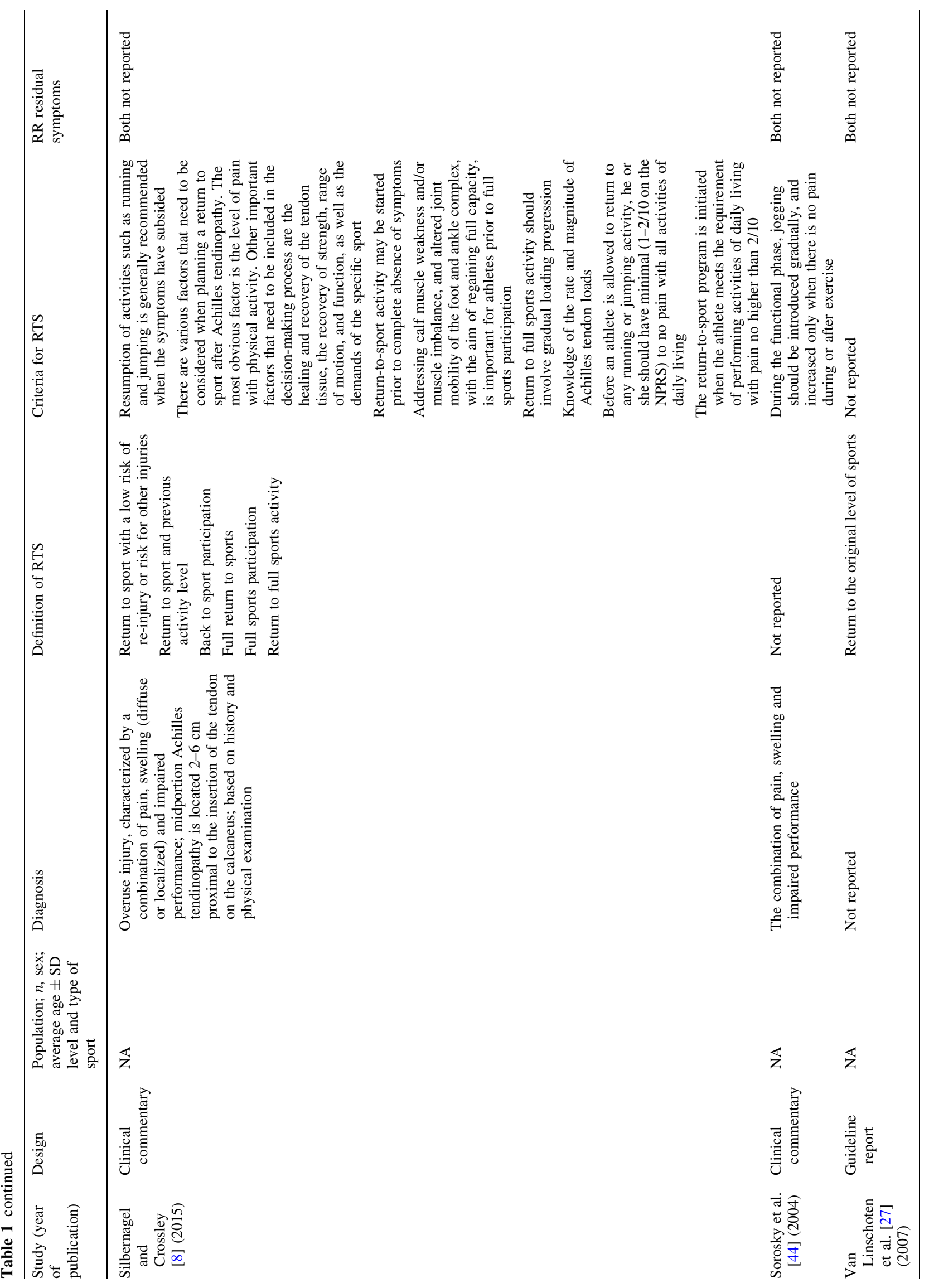




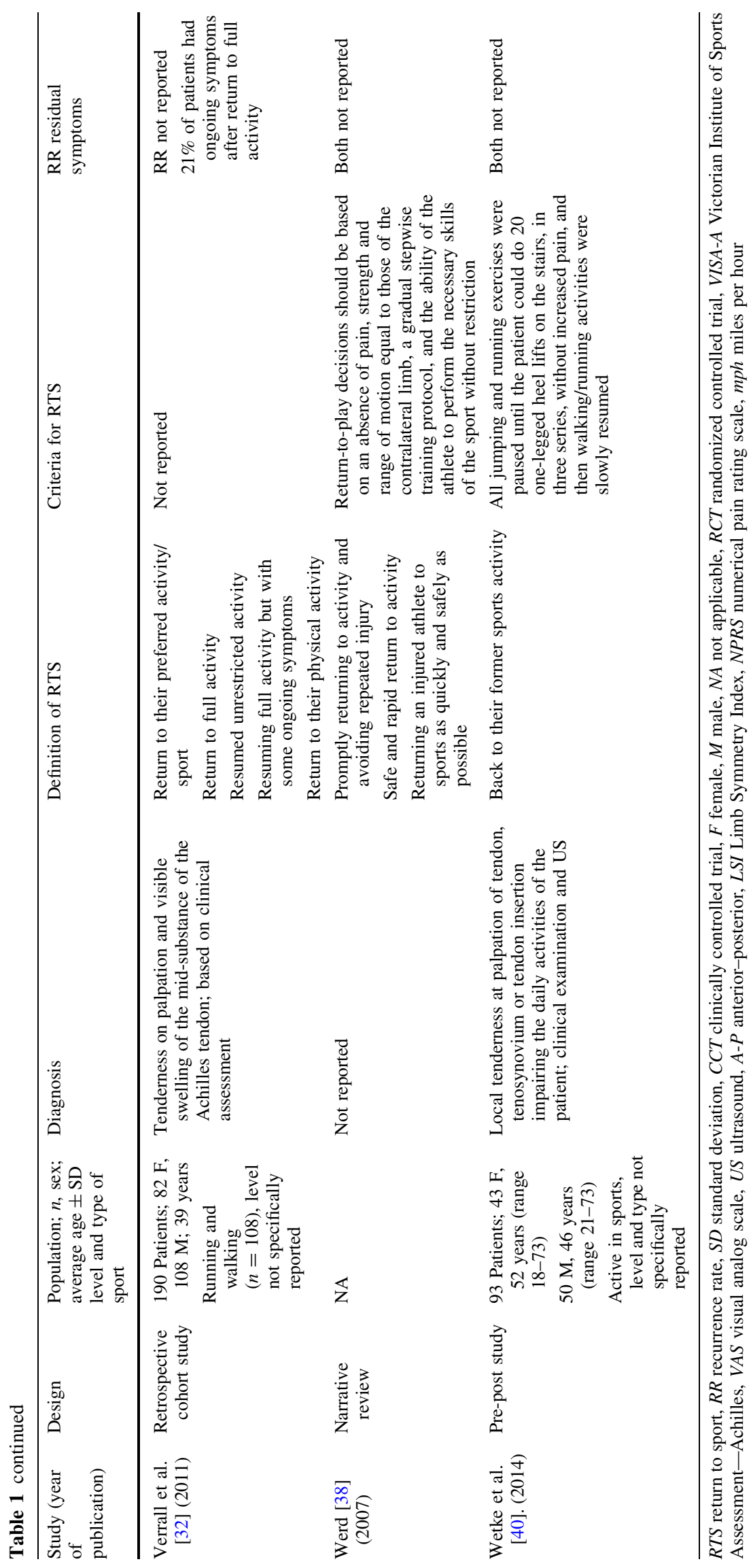


between the two authors. A Cohen's kappa $>0.61$ was considered as substantial agreement.

\subsection{Data Extraction}

Two authors (BHa, AvdB) performed the data extraction from the included studies, using a standardized extraction form. The following relevant data were extracted: (1) first author; (2) year of publication; (3) study design; (4) study population, type and level of sport; (5) definition of the diagnosis of AT; (6) definition of RTS; (7) criteria described for initiation of RTS; and (8) recurrence rate and residual symptoms.

\subsection{Data Analysis}

We searched for definitions of, as well as criteria for, RTS using a content analysis approach [11-13]. This is a qualitative method, aimed at classifying the written material into identified categories in three steps [14]. The first step of content analysis is open coding [15]. Two researchers (BHa, AvdB) independently read through the included studies several times and started to identify provisional labels by making notes in the text indicating text fragments/aspects related to definitions of, or criteria for, RTS. A consensus meeting was conducted to compare the results of this step and discuss potential discrepancies.

The second step is axial coding, which aims to explore the relationships/associations among the provisional labels identified by open coding [15]. Both authors (BHa, AvdB) independently performed the axial coding process, and a consensus meeting was held afterwards to discuss potential discrepancies.

The third step of content analysis is selective coding [15]. During this step, the researchers aimed to develop overarching content categories that serve as umbrella terms for the labels identified during the axial coding phase. In the current review, the selective coding phase resulted in an overview of relevant terms that are used to define RTS after midportion AT, and the criteria that are used for the RTS decision.

\section{Results}

\subsection{Search Results}

The initial search yielded 3862 hits (Fig. 1). After removal of duplicates, 2234 potential articles remained for inclusion. Screening of the titles and abstracts resulted in exclusion of another 2039 articles, leaving 195 articles for full-text assessment. Of these, $10(5 \%)$ could not be obtained in full-text, despite repeated attempts to contact the corresponding author by e-mail or through ResearchGate, and despite attempts to purchase a copy. One hundred and thirty-four studies were excluded after full-text assessment. No consensus was reached on the eligibility of five articles. After consulting our third author $(\mathrm{BHu})$, the studies by Cook et al. [16] and Herrington and McCulloch [17] were included, while three other studies were excluded as they did not provide a definition of, or criteria for, RTS.

Forty-eight articles met our inclusion criteria, but another 13 were excluded as they used a definition that was adopted from other studies. The studies containing the original definition were already included, therefore this resulted in a total of 35 articles that were included in the qualitative content analysis. These 35 studies included 10 randomized controlled trials, two non-randomized controlled trials, four pre-post studies, two retrospective cohort studies, one case series, two case studies, eight narrative reviews, four clinical commentaries, one masterclass report, and one guideline report.

At this stage, Cohen's kappa was 0.69 , indicating substantial agreement [18].

\subsection{Content Analysis}

\subsubsection{Definition}

Of the 35 included studies, $32(91 \%)$ provided one or multiple definitions of RTS for athletes with midportion AT. These definitions were extracted during the open coding phase of the content analysis (Table 1). During the axial coding phase, several categories were formed, which subsequently were grouped into three distinct content categories in the selective coding phase. These content categories were 'pre-injury activity/sports level, with the ability to perform training and matches without limitations', 'absence of pain' and 'recovery' (Fig. 2).

\subsubsection{Reaching Pre-Injury Activity/Sports Level, with the} Ability to Perform Training and Matches Without Limitations The majority of studies used terminology such as 'return to/resume previous activity/sports level' [7, 8, 17, 19-22], 'return to pre-injury activity/sports level' $[6,23-26]$, or 'return to the original activity/sports level' [27-29] to define RTS. This finding was also reported in the included studies as 'return to full (sports) activity' [8, 21, 30-32], 'return to full training schedule without limitations' [28, 33], and 'return to competition' [16, 23].

3.2.1.2 Absence of Pain When defining RTS, a few authors described 'absence of pain' as follows: 'pain-free return to activity' [34], 'return to running without pain' [35], or 'return the patient to the desired level of activity without residual pain' [36]. 


Return to pre-injury activity/sports
level
Return to full activity
Ability to perform pre-injury training
schedule without limitations
Ability to participate in matches
and/or competition
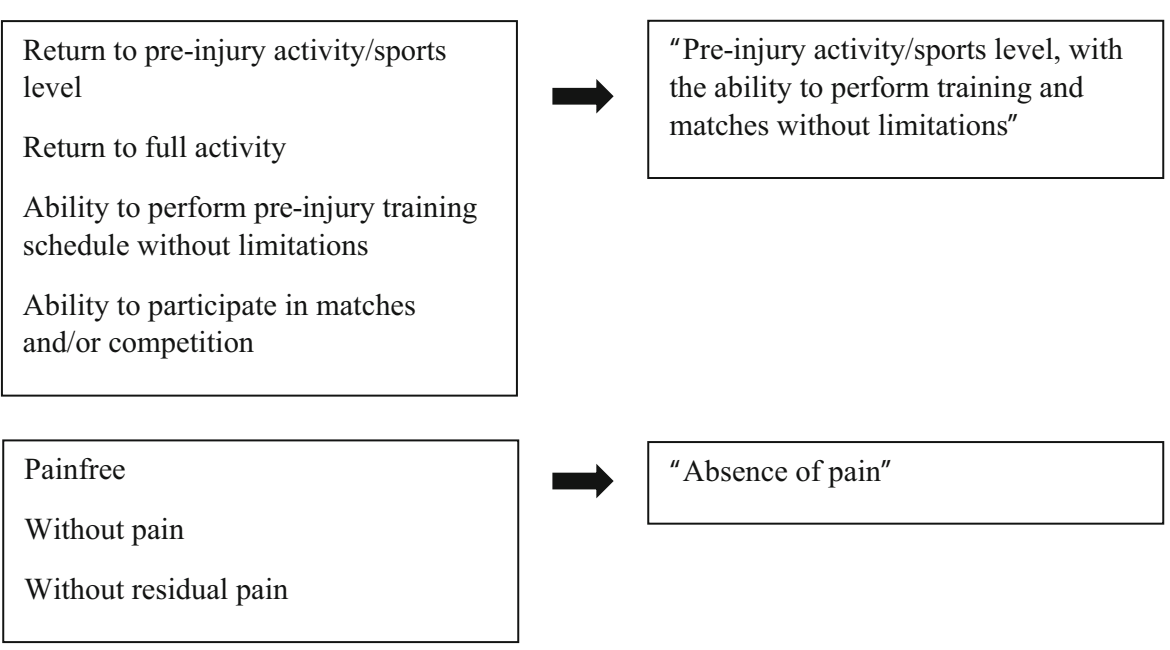

Safe return to activity/sport
Minimizing the risk of re-injury or
other injuries
Rapid recovery
Recovery time as short as possible

Axial coding

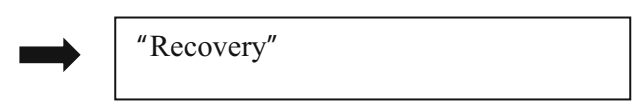

Final content categories established with selective coding

Fig. 2 Axial coding and selective coding of the content analysis for the definition of return to sport after midportion Achilles tendinopathy

3.2.1.3 Recovery In terms of recovery, terminology used to define RTS included 'risk of re-injury' (e.g. 'safe return to sport while minimizing the risk of recurrent injury' [37], 'returning to activity and avoiding repeated injury' [38], and 'time to recovery', which was described as 'swift return' [38] or 'recovery time should be as short as possible' [36].

\subsubsection{Criteria}

Nineteen studies (54\%) reported on one or more criteria for RTS after midportion AT (Table 1). Open coding resulted in different tentative labels, which were categorized during the axial coding phase. The final selective coding phase resulted in eight content categories (Fig. 3).

3.2.2.1 Level of Pain Large variation was seen in the included studies with regard to pain as a criterion for RTS. Some studies reported a complete absence of pain as a criterion for RTS, whereas other studies accepted a certain level of pain. One study reported that pain during sports activities should not exceed $30 \mathrm{~mm}$ on a $0-100 \mathrm{~mm}$ visual analog scale (VAS) [22], while other studies stated that daily activities should be pain-free [35] or with minimal pain (1-2 on a $0-10$ numerical pain rating scale) [8] before RTS can be considered.

3.2.2.2 Level of Functional Recovery Within the included studies, multiple aspects of functional recovery were described as criteria for RTS after AT. Nicola and El Shami reported that return to running should not be considered until one is able to walk comfortably at 4.0 miles per hour (mph) for 10 miles per week [35], whereas Werd stated that "RTS decisions should be based on [...] the ability of the athlete to perform the necessary skills of the sport without restriction" [38].

3.2.2.3 Recovery of Muscular Strength In multiple studies, recovery of muscular strength was described as a criterion for RTS. Silbernagel and Crossley explicitly described that calf muscle weakness should be addressed before RTS [8], but other studies did not explicate the muscle groups that should be addressed.

One study reported a limb symmetry index of $90 \%$ or more as a guideline for RTS [39], while another study stated that recovery of strength to a level equal to the contralateral limb should be achieved [38]. No clear 
No pain during sports activities No severe pain

Pain not exceeding 5 on a $0-10$ visual analog scale

No increase of pain

Minimal residual tenderness

Minimal pain (1-2 on a $0-10$ numerical rating scale) with daily activities

Capable of completing a full practice Able to walk comfortably at $4 \mathrm{mph}$ for 10 miles

Regaining full function

Ability to perform and control necessary sports-specific skills

\section{Recovery of full strength}

Power

No calf muscle weakness

No muscle imbalance

Strength equal to the contralateral limb

Limb symmetry index $\geq 90 \%$

Recovery of full range of motion No altered mobility of foot/ankle Range of motion equal to contralateral limb

\section{Recovery of full endurance}

Completing three series of 20 onelegged heel lifts on the stairs without increased pain

Adequate endurance

Completed rehabilitation program Gradual stepwise training program

Gradual return to sports-specific function

Physical examination

Specific investigations

Demands of the specific sport

Individual goals

Mental aspects

Confidence

Proprioceptive control

Healing and recovery of tendon tissue

Rates and magnitude of Achilles tendon loads

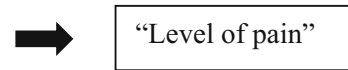

$\Rightarrow \quad$ "Level of functional recovery"

$\Rightarrow \quad$ "Recovery of muscular strength"

$\Rightarrow \quad$ "Recovery of range of motion"

"Level of endurance in the involved limb"

"Medical advice"

"Psychosocial factors"

"Anatomical/physiological properties of the musculotendinous complex"

Final content categories established with selective coding 
4Fig. 3 Axial coding and selective coding of the content analysis for criteria used for return to sport after midportion Achilles tendinopathy

description was given of how muscle strength should be assessed.

3.2.2.4 Recovery of Range of Motion In four studies, range of motion was reported as an RTS criterion for AT, with one study specifying this as 'mobility of the foot and ankle complex' [8]. Werd used the contralateral limb as reference value ('equal to the contralateral limb') [38], whereas other studies provided a more general description, such as 'full range of motion' [37].

\subsubsection{Level of Endurance of the Involved Limb En-} durance was addressed as an RTS criterion for AT in four of the included studies. Wetke et al. stated that jumping and running activities should be ceased until an athlete can perform three sets of 20 one-legged heel lifts on the stairs (without increased pain) [40].

Neither the required level of endurance nor the preferred measurement method were clearly specified in the other studies [23, 37, 41].

3.2.2.6 Medical Advice Several studies described that rehabilitation or a gradual stepwise training protocol should be completed prior to RTS $[8,23,38]$, however, the exact measurement method was not clearly described. In the study by Biedert et al., physical examination and specific tests were also mentioned as RTS criteria for AT [42]; however, these were not further specified.

3.2.2.7 Psychosocial Factors Psychosocial factors as criteria for RTS after AT were mentioned in one study [42]. The authors described that RTS depends on individual goals and mental aspects, but they did not further specify these factors.

3.2.2.8 Anatomical/Physiological Properties of the Musculotendinous Complex In three of the included studies, anatomical/physiological properties of the musculotendinous complex, specified as 'structural healing' [42], 'healing and recovery of the tendon tissue' [8] and 'proprioceptive control' [41], were reported as criteria for RTS after AT. It was not clearly described how these properties were measured, e.g. whether imaging was used to determine the recovery of tendon tissue.

\section{Discussion}

RTS is an important goal for many athletes suffering from midportion AT, and the decision to RTS may be influenced by many factors. This qualitative systematic review aimed to describe how successful RTS after midportion AT is defined, and which criteria are used to support the RTS decision. Of the 35 studies included in this review, $91 \%$ provided a definition, and only $54 \%$ reported criteria for RTS after AT. We found large variation in definitions and criteria for RTS within the different studies. Using a content analysis approach, we aimed to discover content categories that serve as umbrella terms for the definition of, and criteria for RTS after midportion AT.

\subsection{Definitions}

Our content analysis approach identified three distinct content categories used to define successful RTS. Predominantly, we found that 'pre-injury activity/sports level, with the ability to perform training and matches without limitations' seemed to be an important term. We also found that 'absence of pain' and 'recovery' (minimal risk of reinjury or other injuries, and time to recovery) were other important terms used to define RTS after midportion AT.

In a recent consensus statement on RTS after sports injuries in general [4], it was stated that an RTS definition should, at a minimum, describe the type of sport and the sports level that is pursued. Many studies referred to the pre-injury level of sport of the involved athletes, but, unfortunately, this level of sport was often not clearly described. Lack of clear description impedes comparison of pre-injury to post-injury RTS rates; therefore, it will be beneficial to encourage studies to explicitly define the preinjury sport and level of participation of their athletes. Ideally, this should be rated at baseline, or at least early during the intervention, to minimize recall bias of the participants.

Our results further show that, besides the type and level of sport, other relevant terms are also used to define RTS in the current AT literature. These terms were related to symptom level, time to recovery, and risk of re-injury. This implies that merely returning to a certain level of sport is not enough; RTS should also be achieved in a timely manner and with minimal risk of re-injury.

\subsection{Criteria}

In total, $54 \%$ of the included studies described criteria for the RTS decision, but large variation in these criteria was found. Using content analysis, we were able to define eight final content categories: (1) level of pain; (2) level of 
functional recovery; (3) recovery of muscle strength; (4) recovery of range of motion; (5) level of endurance of the involved limb; (6) medical advice; (7) psychosocial factors; and (8) anatomical/physiological properties of the musculotendinous complex.

Many studies described the level of pain as an important criterion for RTS. Seven studies stated that 'no pain' should be present before RTS after midportion AT $[21,31,35,37,38,43,44]$, whereas others used less specific and subjective terms, such as minimal or mild pain/discomfort $[19,25,31,43]$ or no severe pain in the tendon [7, 19]. Silbernagel and Crossley specified that the level of pain during daily activities should not exceed 2 on a $0-10$ numerical pain scale before an athlete is allowed to return to running or jumping activities [8]. Beyer et al. also quantified the maximum level of pain that was allowed before RTS after AT [22], but they specified it as pain during sports activities and the level was slightly higher than the level used by Silbernagel and Crossley (i.e. $30 \mathrm{~mm}$ on a $0-100 \mathrm{~mm}$ VAS).

There is no doubt that pain is an important symptom of AT; in particular, morning pain/stiffness is a hallmark of AT. Morning pain/stiffness is considered as a useful clinical indicator of recovery [16] and has been included as part of the Victorian Institute of Sports AssessmentAchilles (VISA-A) questionnaire, which is considered a valid and reliable tool to evaluate AT symptoms [45]. Remarkably, none of the included studies explicitly described (absence of) morning pain/stiffness as a criterion for RTS. Furthermore, none of the studies used questionnaires such as the VISA-A as a criterion for RTS. It may be useful to investigate the possible role of the VISA-A in the decision to RTS among athletes with midportion AT, and to determine a cut-off score (e.g. $\geq 90$ points [46]) as a required criterion for this decision.

Although many other criteria to support RTS after AT were described in the 35 included studies, it was remarkable that most of these criteria lacked essential information; the relevant body part was not described, no information on the preferred measurement method was given, or clear quantification or cut-off points were lacking. Regarding strength, for instance, studies reported information such as 'balance of strength and flexibility' [41] or 'when full strength has returned' [37]. Only one study explicitly described the relevant muscle group (i.e. calf muscle) [8], and only the study by Silbernagel et al. reported a limb symmetry index of $90 \%$ [39], which is often used as a reference for RTS in clinical practice. Furthermore, the vast majority of studies lacked information on which muscle groups should be tested (e.g. calf muscles, or all muscle groups of the lower extremity), what strength tests should be performed (e.g. isometric or isokinetic), which deficit between the injured and uninjured limb is considered acceptable, and how this could be measured. This lack of information applied to most of the criteria found in this review. This obviously may result in a large variety of measures being used, thereby impeding the clinician's ability to make a well-considered and evidencebased decision on RTS. Additionally, it hampers comparison of RTS rates between different interventions for AT. Thus, we strongly encourage that studies comprehensively describe their criteria for RTS, and define clear cut-off values if possible. Furthermore, it would be of great interest if studies also reported the time to RTS as this is of much importance for clinicians and other stakeholders involved in RTS decision-making.

\subsection{Comparison with Other Findings}

To our knowledge, this is the first systematic review investigating definitions and criteria for RTS in athletes with midportion AT, which limits the comparison with other findings. In the consensus statement on RTS after sports injuries, published by Ardern et al. [4], RTS was described as a process using three elements: (1) return to participation; (2) return to sport; and (3) return to performance. We believe that this categorization of relevant elements has some advantages compared with our findings regarding the definition of RTS. In our review, we found 'pre-injury level of activity/sports, with the ability to perform training and matches without limitations' to be an important term for defining successful RTS, but this appears to refer to the end stage of a rehabilitation process. Using the proposed approach by Ardern et al. [4], RTS is viewed more as a continuum, suggesting that earlier in the process of rehabilitation, athletes may be active in their sport, albeit at a lower level and less intensity.

The consensus statement of Arden et al. further suggested that the rate of RTS after AT varies between 10 and $86 \%$ after 12 weeks of treatment [4]. The authors blame the variety of activity levels for the large variation in RTS rates. At present, we think that the lack of an unambiguous definition may also be responsible for this large variation; if studies interpret RTS differently, this poses difficulty in comparing the success rates for RTS.

Our review attempted to synthesize RTS after temporarily ceasing sports activities. This was in line with the findings of several studies, which reported that up to $72 \%$ of athletes with AT need to cease their sports activities due to ongoing symptoms [29, 32]; however, research has demonstrated that completely ceasing sports activities may not be necessary. This point of view was based on a randomized controlled trial comparing two groups suffering from midportion AT [47]. The first group was allowed to engage in sports activities during the first 6 weeks of rehabilitation, using a pain-monitoring model. They were 
instructed that pain during sports activities should not exceed 5 on a $0-10$ VAS, and that pain and stiffness in the Achilles tendon was not allowed to increase from week to week. The comparison group did not participate in Achilles tendon-loading sport for 6 weeks. As clinical improvement between both groups did not significantly differ, the authors concluded that continuing sports activities during rehabilitation using a pain-monitoring model is justified [47]. Although continuing sports activities using a painmonitoring model may have advantages over temporary interruption (e.g. retaining tendon loading capacity and a positive effect on general health and psychological wellbeing), this decision should be made on an individual basis and should consider factors such as level of symptoms and psychological factors [48].

In a recent review of RTS after a rupture of the Achilles tendon [49], the authors concluded that $80 \%$ (range $18.6-100 \%)$ of athletes returned to sport approximately 6 months after the injury. However, interestingly, both rate and time differed between the included studies that clearly described definitions and measures of return to play, and those studies that did not provide a description of how RTS was assessed [49]. These findings are in line with our results, namely that there was a large variation in how RTS is defined, and many studies did not provide sufficient information on the type of measures that should be used to support the RTS decision. Therefore, we strongly advise both clinicians and researchers to achieve consensus, not only on a uniform definition for RTS after AT but also to define what measures (physical tests, performance tests, questionnaires, psychological factors, imaging) should be included in order to make the RTS decision process more efficient and successful. As many criteria are inter-related, it would be worthwhile to consider grouping them together with respect to clinical purpose. In future research, this may be addressed by performing a Delphi consensus strategy, similar to what was recently done for RTS after hamstring injuries [50].

\subsection{Strengths and Limitations}

A strength of this review is that it was conducted in accordance with the PRISMA guidelines, which enhances its methodological quality. Additionally, we made no restrictions on study design in our selection criteria. While this may also be regarded as a limitation of the study, we feel that this decision maximized the chance of finding relevant literature on RTS after AT.

Our study also has some limitations that need to be addressed. First, a considerable proportion of studies $(n=10)$ could not be obtained in full text, despite serious efforts to contact the corresponding author of these studies (e-mail, ResearchGate) to obtain a copy. These studies may have used different definitions and/or criteria for RTS, which could obviously have influenced our results. Second, although we did not place limitations on study design, we only included studies investigating the effects of physiotherapeutic interventions. Therefore, we do not know whether studies on medication, injection, or operative treatments used different definitions and/or criteria.

\section{Conclusions}

This qualitative systematic review revealed a large variation within AT research in how RTS is defined and which criteria should be used to support the RTS decision. This limits the clinician's ability to make a well-considered RTS decision, and also hampers the comparison of RTS rates in different intervention studies. Using a content analysis approach, this systematic review showed that RTS may be defined according to the pre-injury level of sports (including both training and matches), but also with terms related to the absence of pain and recovery.

Currently, RTS decisions for midportion AT seem to be based on multiple criteria, which are all related to level of pain, level of functional recovery, muscular strength, range of motion, endurance, medical advice, psychosocial factors, and anatomical/physiological properties of the Achilles tendon. It was remarkable that, for most of the criteria we identified, no clear operationalization was given, which limits their practical usability. Therefore, there is an urgent need for future research aiming to reach consensus on how RTS after midportion AT should be defined, and what criteria should be used to support the decision on RTS.

\section{Compliance with Ethical Standards}

Funding No sources of funding were used to assist in the preparation of this article.

Conflicts of interest Bas Habets, Anke van den Broek, Bionka Huisstede, Frank Backx and Robert van Cingel declare that they have no conflicts of interest relevant to the content of this review.

Open Access This article is distributed under the terms of the Creative Commons Attribution 4.0 International License (http:// creativecommons.org/licenses/by/4.0/), which permits unrestricted use, distribution, and reproduction in any medium, provided you give appropriate credit to the original author(s) and the source, provide a link to the Creative Commons license, and indicate if changes were made.

\section{References}

1. Kvist M. Achilles tendon injuries in athletes. Sports Med. 1994;18(3):173-201. 
2. Gajhede-Knudsen M, Ekstrand J, Magnusson H, Maffulli N. Recurrence of Achilles tendon injuries in elite male football players is more common after early return to play: an 11-year follow-up of the UEFA Champions League injury study. Br J Sports Med. 2013;47(12):763-8.

3. Silbernagel KG, Thomeé R, Eriksson BI, Karlsson J. Full symptomatic recovery does not ensure full recovery of muscletendon function in patients with Achilles tendinopathy. $\mathrm{Br} \mathbf{J}$ Sports Med. 2007;41(4):276-80.

4. Ardern CL, Glasgow P, Schneiders A, Witvrouw E, Clarsen B, Cools A, et al. 2016 Consensus statement on return to sport from the First World Congress in Sports Physical Therapy. Bern. Br J Sports Med. 2016;50(14):853-64.

5. Habets B, Van Cingel REH. Eccentric exercise training in chronic mid-portion Achilles tendinopathy: a systematic review on different protocols. Scand J Med Sci Sports. 2015;25(1):3-15.

6. Roos EM, Engstrom M, Lagerquist A, Soderberg B. Clinical improvement after 6 weeks of eccentric exercise in patients with mid-portion Achilles tendinopathy: a randomized trial with 1-year follow-up. Scand J Med Sci Sports. 2004;14(5):286-95.

7. Mafi N, Lorentzon R, Alfredson H. Superior short-term results with eccentric calf muscle training compared to concentric training in a randomized prospective multicenter study on patients with chronic Achilles tendinosis. Knee Surg Sports Traumatol Arthrosc. 2001;9(1):42-7.

8. Silbernagel KG, Crossley KM. A proposed return-to-sport program for patients with midportion Achilles tendinopathy: rationale and implementation. $\mathbf{J}$ Orthop Sports Phys Ther. 2015;45(11):876-86.

9. Maffulli N, Khan KM, Puddu G. Overuse tendon conditions: time to change a confusing terminology. Arthroscopy. 1998;14(8):840-3.

10. van der Horst N, van de Hoef S, Reurink G, Huisstede B, Backx F. Return to play after hamstring injuries: a qualitative systematic review of definitions and criteria. Sports Med. 2016;46(6):899-912.

11. Cavanagh S. Content analysis: concepts, methods and applications. Nurse Res. 1997;4(3):5-16.

12. Krippendorff K. Content analysis: an introduction to its methodology. 2nd ed. Thousand Oaks: Sage Publications; 2004.

13. Elo $\mathrm{S}, \mathrm{Kyngas} \mathrm{H}$. The qualitative content analysis process. J Adv Nurs. 2008;62(1):107-15.

14. Moretti F, van Vliet L, Bensing J, Deledda G, Mazzi M, Rimondini $\mathrm{M}$, et al. A standardized approach to qualitative content analysis of focus group discussions from different countries. Patient Educ Couns. 2011;82(3):420-8.

15. Corbin J, Strauss A. Basics of qualitative research: techniques and procedures for developing grounded theory. 4th ed. Thousand Oaks: Sage Publications; 2015.

16. Cook JL, Khan KM, Purdam C. Achilles tendinopathy. Man Ther. 2002;7(3):121-30.

17. Herrington $\mathrm{L}, \mathrm{McCulloch} \mathrm{R}$. The role of eccentric training in the management of Achilles tendinopathy: a pilot study. Phys Ther Sport. 2007;8(4):191-6.

18. Landis JR, Koch GG. The measurement of observer agreement for categorical data. Biometrics. 1977;33(1):159-74.

19. Fahlströhm M, Jonsson P, Lorentzon R, Alfredson H. Chronic Achilles tendon pain treated with eccentric calf-muscle training. Knee Surg Sports Traumatol Arthrosc. 2003;11(5):327-33.

20. Alfredson H, Cook J. A treatment algorithm for managing Achilles tendinopathy: New treatment options. Br J Sports Med. 2007;41(4):211-6.

21. Rompe JD, Furia J, Maffulli N. Eccentric loading versus eccentric loading plus shock-wave treatment for midportion Achilles tendinopathy: a randomized controlled trial. Am J Sports Med. 2009;37(3):463-70.
22. Beyer R, Kongsgaard M, Hougs Kjaer B, Ohlenschlaeger T, Kjaer M, Magnusson SP. Heavy slow resistance versus eccentric training as treatment for Achilles tendinopathy: a randomized controlled trial. Am J Sports Med. 2015;43(7):1704-11.

23. Kountouris A, Cook J. Rehabilitation of Achilles and patellar tendinopathies. Best Pract Res Clin Rheumatol. 2007;21(2):295-316.

24. Paavola M, Kannus P, Paakkala T, Pasanen M, Jarvinen M. Longterm prognosis of patients with Achilles tendinopathy. an observational 8-year follow-up study. Am J Sports Med. 2000;28(5):634-42.

25. Petersen W, Welp R, Rosenbaum D. Chronic Achilles tendinopathy: a prospective randomized study comparing the therapeutic effect of eccentric training, the AirHeel brace, and a combination of both. Am J Sports Med. 2007;35(10):1659-67.

26. Giombini A, Di Cesare A, Casciello G, Sorrenti D, Dragoni S, Gabriele P. Hyperthermia at $434 \mathrm{MHz}$ in the treatment of overuse sport tendinopathies: a randomised controlled clinical trial. Int $\mathbf{J}$ Sports Med. 2002;23(3):207-11.

27. van Linschoten $\mathrm{R}$, den Hoed PT, de Jongh AC. Guideline 'Chronic Achilles tendinopathy, in particular tendinosis, in sportsmen/sportswomen'. Ned Tijdschr Geneeskd. 2007;151(42):2319-24 (in Dutch)

28. Lakshmanan P, O'Doherty DP. Chronic Achilles tendinopathy: treatment with extracorporeal shock waves. Foot Ankle Surg. 2004;10(3):125-30.

29. de Vos R, Weir A, Visser RJ, de Winter T, Tol JL. The additional value of a night splint to eccentric exercises in chronic midportion Achilles tendinopathy: a randomised controlled trial. Br J Sports Med. 2007;41(7):e5.

30. Chazan IM. Achilles tendinitis part II: clinical examination, differential diagnosis, and approaches to management. J Man Manipul Ther. 1998;6(2):70-7.

31. Rompe JD, Nafe B, Furia JP, Maffulli N. Eccentric loading, shock-wave treatment, or a wait- and-see policy for tendinopathy of the main body of tendo Achillis: a randomized controlled trial. Am J Sports Med. 2007;35(3):374-83.

32. Verrall G, Schofield S, Brustad T. Chronic Achilles tendinopathy treated with eccentric stretching program. Foot Ankle Int. 2011;32(9):843-9.

33. Barry M. Bringing Achilles tendinopathy to heel. Nursing. 2010;40(10):30-3.

34. McShane JM, Ostick B, McCabe F. Noninsertional Achilles tendinopathy: pathology and management. Curr Sports Med Rep. 2007;6(5):288-92.

35. Nicola TL, El Shami A. Rehabilitation of running injuries. Clin Sports Med. 2012;31(2):351-72.

36. Paavola M, Kannus $P$, Järvinen TA, Khan K, Józsa L, Järvinen M. Achilles tendinopathy. J Bone Joint Surg. 2002;84(11):2062-76.

37. Chinn L, Hertel J. Rehabilitation of ankle and foot injuries in athletes. Clin Sports Med. 2010;29(1):157-67.

38. Werd MB. Achilles tendon sports injuries: a review of classification and treatment. J Am Podiatr Med Assoc. 2007;97(1):37-48.

39. Silbernagel KG, Brorsson A, Lundberg M. The majority of patients with Achilles tendinopathy recover fully when treated with exercise alone: a 5-year follow-up. Am J Sports Med. 2011;39(3):607-13.

40. Wetke E, Johannsen F, Langberg H. Achilles tendinopathy: a prospective study on the effect of active rehabilitation and steroid injections in a clinical setting. Scand J Med Sci Sports. 2015;25(4):e392-9.

41. Chessin M. Achilles tendinosis stopping the progression to disability. J Dance Med Sci. 2012;16(3):109-15. 
42. Biedert RM, Hintermann B, Hörterer H, Müller AE, Warnke K, Friederich N, et al. Return to sport after injuries and operative treatment. Sport Orthop Sport Traumatol. 2006;22(4):249-54.

43. Alfredson H, Pietila T, Jonsson P, Lorentzon R. Heavy-load eccentric calf muscle training for the treatment of chronic Achilles tendinosis. Am J Sports Med. 1998;26(3):360-6.

44. Sorosky B, Press J, Plastaras C, Rittenberg J. The practical management of Achilles tendinopathy. Clin J Sport Med. 2004;14(1):40-4.

45. Robinson JM, Cook JL, Purdam C, Visentini PJ, Ross J, Maffulli $\mathrm{N}$, et al. The VISA-A questionnaire: a valid and reliable index of the clinical severity of achilles tendinopathy. Br J Sports Med. 2001;35(5):335-41.

46. Iversen JV, Bartels EM, Langberg H. The Victorian Institute of Sports Assessment-Achilles questionnaire (VISA-A): a reliable tool for measuring Achilles tendinopathy. Int J Sports Phys Ther. 2012;7(1):76-84.

47. Silbernagel KG, Thomeé R, Eriksson BI, Karlsson J. Continued sports activity, using a pain-monitoring model, during rehabilitation in patients with Achilles tendinopathy: a randomized controlled study. Am J Sports Med. 2007;35(6):897-906.

48. Mallows A, Debenham J, Walker T, Littlewood C. Association of psychological variables and outcome in tendinopathy: a systematic review. Br J Sports Med. 2017;51(9):743-8.
49. Zellers JA, Carmont MR, Gravare Silbernagel K. Return to play post-Achilles tendon rupture: a systematic review and metaanalysis of rate and measures of return to play. Br J Sports Med. 2016;50(21):1325-32.

50. van der Horst N, Backx F, Goedhart EA, Huisstede BM. HIPSDelphi Group. Return to play after hamstring injuries in football (soccer): a worldwide Delphi procedure regarding definition, medical criteria and decision-making. $\mathrm{Br} \mathrm{J}$ Sports Med. 2017;51(22):1583-91.

51. Ammendolia A, Cespites M, Iocco M. Topical use of aloe gel and low-level laser therapy in overuse tendinitis of elite volleyball players: a randomized controlled trial. Sport Sci Health. 2016;12:209-13.

52. Dijkstra HJW, Van Enst GC. The therapeutic value of G-brace in the treatment of chronic Achilles tendinosis: a pilot study. Geneeskunde Sport. 2003;36(5):137-40.

53. Langberg $\mathrm{H}$, Ellingsgaard $\mathrm{H}$, Madsen $\mathrm{T}$, Jansson J, Magnusson SP, Aagaard P, et al. Eccentric rehabilitation exercise increases peritendinous type I collagen synthesis in humans with Achilles tendinosis. Scand J Med Sci Sports. 2007;17(1):61-6.

54. Ross G, Macfarlane C, Vaughan B. Combined osteopathy and exercise management of Achilles tendinopathy in an athlete: a case report. J Sports Med Phys Fitness. 2018;58(1-2):106-12. 